
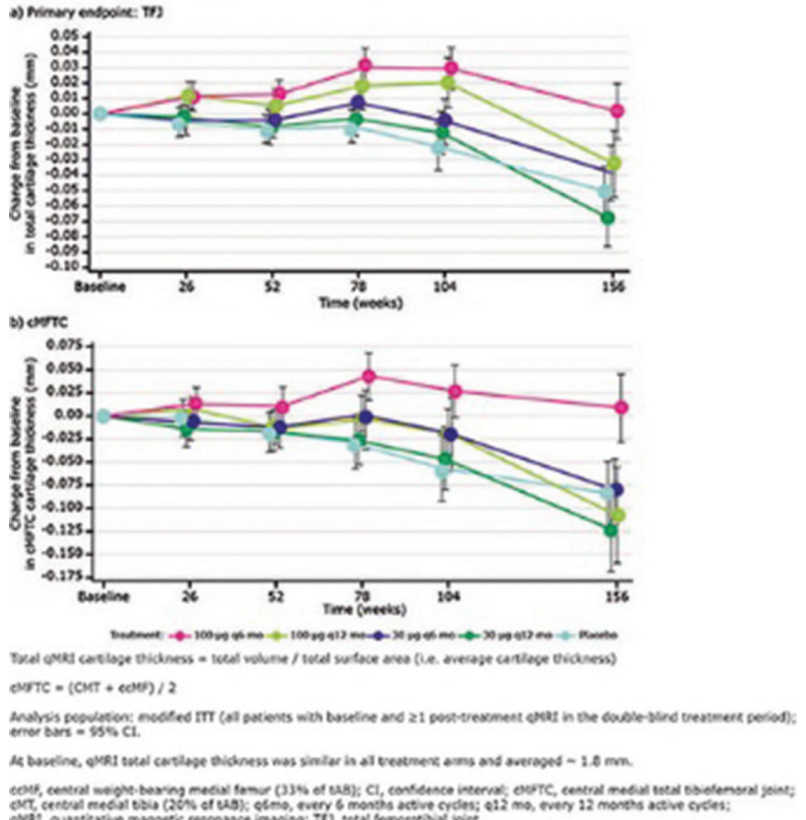

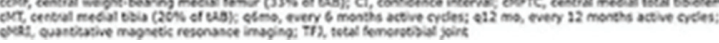

Abstract OP0059 - Figure 1Mean change from baseline in cartilage thickness (by qMRI) over 3 years in: a) and b)

Conclusions: The 3 year results of the FORWARD study are consistent with the 2 year results: although cartilage thickness declined in all treatment groups between Year 2 and 3, the difference at Year 2 with sprifermin $100 \mu \mathrm{g}$ vs PBO was maintained up to Year 3. Based on qMRI sprifermin is effective at increasing cartilage thickness in a dose-dependent manner in knee OA patients, and has an acceptable safety profile.

Disclosure of Interest: M. Hochberg Consultant for: Bioiberica SA, Bristol Myers Squibb, EMD Serono, Inc., Galapagos, IBSA Biotechniq SA, Novartis Pharma AG, Pfizer Inc., Plexxikon, Samumed LLC, Theralogix LLC and TissueGene Inc, A. Guermazi Consultant for: OrthoTrophix, GE Healthcare, Merck Serono, AstraZeneca, Sanofi, TissueGene and Pfizer, Employee of: Boston Imaging Core Lab, LLC, H. Guehring Employee of: Merck KGaA, A. Aydemir Employee of: EMD Serono, Inc., S. Wax Employee of: EMD Serono, Inc., P. Fleuranceau-Morel Employee of: EMD Serono, Inc., A. Reinstrup Bihlet Shareholder of: Nordic Bioscience, Employee of: Nordic Bioscience, I. Byrjalsen Employee of: Nordic Bioscience, J. Ragnar Andersen Shareholder of: Nordic Bioscience, Employee of: Nordic Bioscience, F. Eckstein Shareholder of: Chondrometrics GmbH, Consultant for: Merck KGaA, Samumed LLC, Abbvie, Bioclinica, TissueGene, Servier and Roche, Employee of: Chondrometrics GmbH

DOI: 10.1136/annrheumdis-2018-eular.2181

\section{OP0060 KNEE JOINT DISTRACTION COMPARED WITH HIGH TIBIAL OSTEOTOMY AND TOTAL KNEE ARTHROPLASTY: TWO-YEAR CLINICAL, STRUCTURAL, AND BIOMARKER OUTCOMES}

M. Jansen ${ }^{1}$, N. Besselink ${ }^{1}$, R. Van Heerwaarden ${ }^{2}$, R. Custers ${ }^{3}$, J.-T. Van der Woude $^{1}$, K. Wiegant ${ }^{1}$, S. Spruijt ${ }^{2}$, P. Emans ${ }^{4}$, P. Van Roermund ${ }^{3}$, S. Mastbergen ${ }^{1}$ F. Lafeber ${ }^{1} .{ }^{1}$ Rheumatology and Clinical Immunology, University Medical Center Utrecht, Utrecht, ${ }^{2}$ Department of Orthopedic Surgery, Maartenskliniek Woerden, Woerden; ${ }^{3}$ Department of Orthopedics, University Medical Center Utrecht, Utrecht, ${ }^{4}$ Department of Orthopedics, Maastricht University Medical Center, Maastricht, Netherlands

Background: Knee joint distraction (KJD) is a new joint-preserving surgery technique that, like high tibial osteotomy (HTO), aims to delay total knee arthroplasty (TKA) especially in younger patients with knee osteoarthritis (OA). One year after treatment, KJD demonstrated similar beneficial outcomes compared to HTO and compared to TKA. ${ }^{12}$

Objectives: To compare radiographic joint space width and clinical outcome over two-years for KJD vs TKA and for KJD vs HTO and to additionally study KJD cartilage repair by evaluation of systemic collagen type II markers.

Methods: End-stage knee OA patients considered for TKA were randomised to KJD $\left(n=20 ; K_{J} D_{\text {TKA }}\right)$ or TKA $(n=40)$. Medial compartmental knee OA patients with a varus deviation of $<10^{\circ}$ considered for opening wedge $\mathrm{HTO}$ were randomised to KJD $\left(n=23 ; K J D_{\text {Нто }}\right)$ or $H T O(n=46)$. Distraction surgery was performed by use of two external fixators with built in springs, placed lateral and medial of the knee joint. The knee was distracted $5 \mathrm{~mm}$ for 6 weeks and weight-bearing was encouraged.

WOMAC questionnaires (100 best) and VAS pain scores ( 0 best) were assessed at baseline (0), 3, 6, 12, 18 and 24 months. In the KJD groups, serum PIIANP and urine CTXII levels, as markers for collagen type II synthesis and breakdown, were determined over time. Normalised $Z$-indexes were calculated $\left(Z_{\text {index }}=Z_{\text {PIIANP }}-\right.$ $Z_{\text {СTXIII }}$ to express net collagen type II synthesis. The minimum and mean joint space width (JSW) of the most affected compartment (MAC) were measured with KIDA software on standardised radiographs taken at 0,12 and 24 months.

Results: Of the 129 included patients, 1, 6, 3, and 5 patients were lost in the KJD TKA, TKA, KJD ${ }_{\text {HTO }}$, and HTO group respectively, for various reasons. Oneyear structural and clinical outcomes were statistically significantly improved as reported before, and these beneficial effects sustained for at least two years after treatment when compared to baseline (figure $1 \mathrm{~A}-\mathrm{C}$ ).

At 24 months, there were no significant differences between the $K_{J D} D_{\text {HTO }}$ and HTO groups (all $p>0.25$ ) and between the KJD $\mathrm{TKA}_{\text {TKA }}$ and TKA group, except for VAS pain score in favour of TKA at 24 months $(p=0.037$; figure $1 B)$.

Compared to baseline, the ratio of synthesis over breakdown of collagen type II biomarkers (figure 1D) was significantly decreased at 3 months $(-0.45 \pm 0.20$; $\mathrm{p}=0.032$ ) after which this reversed towards an increase over time (at 24 months $+0.59 \pm 0.19 ; p=0.004$ ).
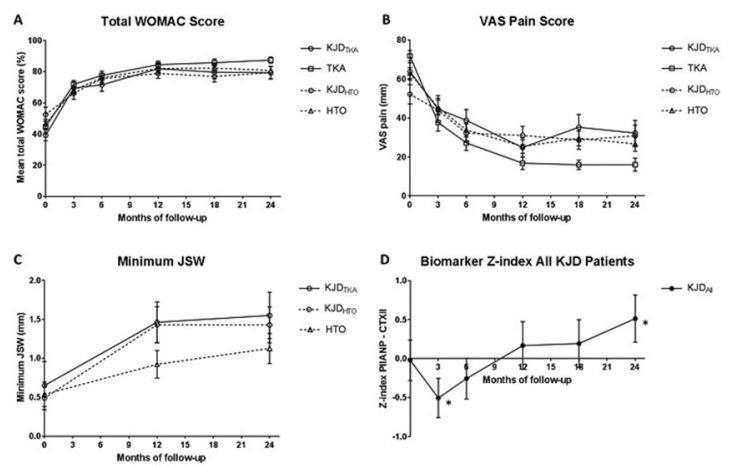

Abstract OP0060 - Figure 1 Change in A) total WOMAC score and B) VAS pain scoe afte treatment with knee joint distraction (KJD), total knee arthroplasty (TKA) and high tibial osteotomy (HTO). KJD patients are divided in KJD TKA (from the trial including patients for KJD and TKA) and KJD HTO (from the trial including patients for HTO and TKA). C) change in minimum joint space width (JSW) measured on radiographs after KJD and HTO. D) biomarkerZ-index changes for all KJD patients (KJD TKA and KID HTO $_{\text {combined) after }}$ treatment. $Z_{\text {index }}=Z_{\text {PIIANP }}-Z_{\text {CTXIII }}$, where the $Z$-values for both biomarkers are relative to baseline and $\left(^{*}\right)$ indicates statistically significant changes compared to baseline. In all graphs, the mean values \pm SEM are given.

Conclusions: Sustained improvement of clinical benefit and increase in JSW after KJD is demonstrated for over 2 years of follow-up in case of treatment of patients with medial compartmental knee OA indicated for HTO or patients with end-stage knee OA indicated for TKA. The structural cartilage repair observed on radiographs is supported by a beneficial change in systemic biomarkers for collagen type II. For the HTO-indicated population, results of KJD patients were similar to those of HTO. For the TKA-indicated patients, TKA appeared to result in a slightly better clinical outcome, however at the expense of the native knee joint.

\section{REFERENCES:}

[1] vd Woude JA, et al. Bone Joint J 2017;99-B(1):51-58.

[2] vd Woude JA, et al. Knee Surg Sports Traumatol Arthrosc 2017;25 (3):876-886

Disclosure of Interest: None declared DOI: 10.1136/annrheumdis-2018-eular.5846

\begin{tabular}{|l|l|}
\hline OP0061 & TREATMENT OF KNEE OSTEOARTHRITIS WITH \\
SM04690 IMPROVED WOMAC A1 'PAIN ON WALKING' - \\
RESULTS FROM A 52 WEEK, RANDOMISED, DOUBLE- \\
BLIND, PLACEBO-CONTROLLED, PHASE 2 STUDY OF A \\
NOVEL, INTRA-ARTICULAR, WNT PATHWAY INHIBITOR
\end{tabular}

S. Kennedy ${ }^{1}$, H. Ghandehari ${ }^{1}$, C. Swearingen ${ }^{1}$, J. Tambiah ${ }^{1}$, M. Hochberg ${ }^{2}$. ${ }^{1}$ Samumed, LLC, San Diego; ${ }^{2}$ University of Maryland School of Medicine, Baltimore, USA

Background: Knee osteoarthritis $(\mathrm{OA})$ is characterised by pain, disability and joint deformity due to articular cartilage degradation and bone remodelling. Wnt 